\title{
Bayesian Prediction based on an Adaptive Type-II Progressive Censored Data from the Generalized Exponential Distribution
}

\author{
M. M. Mohie El-Din \\ Department of Mathematics \\ Faculty of Science \\ Al-Azhar University \\ Egypt
}

\author{
M. M. Amein \\ Department of Mathematics \\ Faculty of Science \\ Al-Azhar University \\ Egypt
}

\author{
A. R. Shafay \\ Department of Mathematics \\ Faculty of Science \\ Fayoum University \\ Egypt
}

\author{
S. Mohamed \\ Department of Mathematics \\ Faculty of Science \\ Fayoum University \\ Egypt
}

\begin{abstract}
In this paper, based on an observed adaptive Type-II progressively censored sample from the generalized exponential distribution, the problem of predicting the order statistics from a future unobserved sample from the same distribution is discussed. The description of the model of the adaptive Type-II progressively censored sample from the generalized exponential distribution is presented. Also, Markov chain Monte Carlo method is applied to construct the Bayesian prediction intervals of the order statistics from a future sample from the same distribution. Finally, results from simulation studies assessing the performance of our proposed method are included and then an illustrative example using real data set is presented for illustrating all the inferential procedures developed here.
\end{abstract}

\section{General Terms}

AMS 2000 Subject Classification: Primary 62G30; Secondary 62F15

\section{Keywords}

Adaptive Type-II progressive censored scheme, Bayesian prediction, Generalized exponential distribution, Markov chain Monte Carlo technique

\section{INTRODUCTION}

There are several types on life testing in which units are removed or lost from the experiment before the failure. Data which obtained from such experiment are called censored data. The most common reason for censoring is reducing the total time of the test and the associated cost and effort. Also, a censoring scheme which can balance between, total time spent for the experiment, number of units placed on the experiment and the efficiency of statistical inference based on the results of the experiment, is desirable.

Type-I (time) and Type-II (failure) censoring schemes are the most common censoring schemes. Under Type-I censoring scheme, the life testing experiment will be stopped at a pre-fixed time $T$, while under Type-II censoring scheme, the life testing experiment will be terminated at the time when the $r^{t h}$ failure is observed. Progressive Type-II censoring scheme is a generalization of Type-II censoring scheme, where $n$ units are placed on the life testing experiment and only $m$ failures are going to be observed. At the first observed failure, $R_{1}$ of the surviving units are randomly selected and removed. When the second failure is observed, $R_{2}$ of the surviving units are randomly selected and removed. The experiment will be terminated at when the $m^{t h}$ failure is observed and all remaining $R_{m}=n-R_{1}-R_{2}-\ldots-R_{m-1}-m$ surviving units are removed. We denote a progressively Type-II censored sample by $X_{1: m: n}<X_{2: m: n}<\ldots<X_{m: m: n}$. For extensive reviews of the literature on progressive censoring, readers may refer to Balakrishnan and Aggarwala (2000), Balakrishnan (2007), Ng and Chan (2007), and Mohie El-Din and Shafay (2013).

Recently, Ng et al (2009) have suggested an adaptive Type-II progressive censoring which is a mixture of Type-I and Type-II progressive censoring schemes. In this censoring scheme, we allow $R_{1}-R_{2}-\cdots-R_{m}$ to depend on the failure times so that the effective sample size is always $m$ which is fixed in advance. A properly planned adaptive progressively censored life testing experiment can save both the total test time and the cost induced by failure of the units and increase the efficiency of statistical analysis. This censoring scheme can be described as follows: Consider $n$ identical units under observation in a life-testing experiment and suppose the experimenter provides a time $T$, which is an ideal total test time, but we may allow the experiment to run over time $T$. If the $m^{t h}$ progressively censored observed failure occurs before time $T$ (i.e. $X_{m: m: n}<T$ ), the experiment terminates at the time $X_{m: m: n}$ (progressive Type-II censoring case); see Fig. 1 .

Otherwise, once the experimental time passes time $T$ but the number of observed failures has not reached $m$, we would want to terminate the experiment as soon as possible. Therefore, we should leave as many surviving items on the test as possible. Suppose $J$ is the number of failures observed before time $T$, i.e. $X_{J: m: n}<$ $T<X_{J+1: m: n}, J=0,1, \ldots, m$, where $X_{0: m: n} \equiv 0$ and $X_{m+1: m: n} \equiv \infty$. After the experiment passed time $T$, we set $R_{J+1}=\ldots=R_{m-1}=0$ and $R_{m}=n-m-\sum_{i=1}^{J} R_{i}$; see Fig.2.

This formulation leads us to terminate the experiment as soon as possible if the $(J+1)^{t h}$ failure time is greater than $T$ for $J+1<m$. The value of $T$ plays an important role in the determination of the values of $R_{i}$ and also as a compromise between a shorter experimental time and a higher chance to observe extreme 


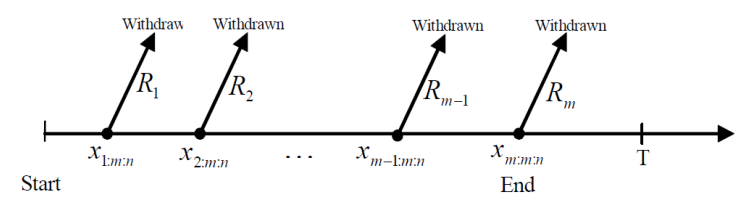

Fig. 1. experiment terminate before time $T$.

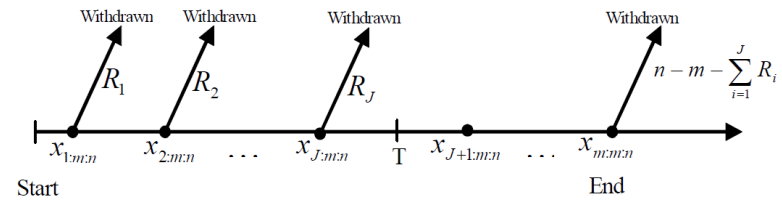

Fig. 2. experiment terminate after time $T$.

failures. One extreme case is when $T \rightarrow \infty$, which means time is not the main consideration for the experimenter, then we will have a usual progressive Type-II censoring scheme with the pre-fixed progressive censoring scheme $\left(R_{1}, \ldots, R_{m}\right)$. Another extreme case can occur when $T=0$, which means we always want to end the experiment as soon as possible, then we will have $R_{1}, \ldots, R_{m-1}=0$ and $R_{m}=n-m$ which results in the conventional Type-II censoring scheme. For extensive reviews of the literature on the adaptive Type-II progressive censoring scheme, readers may refer to Cramer and Iliopoulos (2010), Mahmoud et al. (2013), and Amein (2016, 2017).

Statistical prediction can be applied in many domains such as, quality control, forecasting, marketing, engineering, industry, business, reliability and many others. In each of these domains, it can be used for planning purposes (predict the total medical cost of a population, predict a future number of insurance claims), for process monitoring (predict the number of nuclear scrams in a power plant), or for decision making. There is a large amount of literature describing various statistical prediction applications and methods. Some of these application may be found in Nelson (1982), Devroy and Gyorfi (1985), Bernardo (1988), Brown and Makalainen (1992). Predicting an observable in the future sample depends on the used sampling scheme. Statistical prediction is provided some estimates (point or interval) for future observations based on the results of past (informative) sample. The predictive intervals are the most familiar forms of prediction. They differ significantly from the confidence intervals and tolerance regions, which deal mainly with the unknown population parameters. A predictive interval is an interval that uses the results of an observed sample to contain the results of a future (unobserved) sample with a specified probability.

Recently, Mohie El-Din et al. (2017) have considered the adaptive Type-II progressive censoring scheme and used the maximum likelihood and Bayesian methods to calculate the point estimators and the approximate confidence intervals for the unknown parameters as well as the reliability and hazard rate functions of the generalized exponential distribution. In this paper, based on an observed adaptive Type-II progressively censored sample from the generalized exponential distribution, the problem of predicting the order statistics from a future sample from the same distribution is discussed. the rest of paper is organized as follows. In Section 2, the description of the model of the adaptive Type-II progressively censored sample from the generalized exponential distribution is presented. In Section 3, Markov chain Monte Carlo (MCMC) method is applied to construct the Bayesian prediction intervals of the order statistics from a future sample from the same distribution. Finally, in Section 4, results from simulation studies assessing the performance of our proposed method are included and then an illustrative example using real data set is presented for illustrating all the inferential procedures developed here.

\section{THE MODEL DESCRIPTION}

Suppose $n$ items on a life-testing experiment and the experimenter provides a time $T$. If the failure times of the items are from a continuous population with cumulative distribution function (CDF) $F(x)$ and probability density function (PDF) $f(x)$. Then, the joint density function of the adaptive Type-II progressively censored sample $\underline{X}=\left(X_{1, m, n}, \ldots, X_{m, m, n}\right)$ with censoring scheme $\underline{R}=$ $\left(R_{1}, \ldots, R_{m}\right)$ is then given by (see $\mathrm{Ng}$ et al., 2009)

$$
\begin{aligned}
f\left(x_{1}, \cdots, x_{m}\right)= & d_{J}\left(\prod_{i=1}^{m} f\left(x_{i: m: n}\right)\right)\left(\prod_{i=1}^{J}\left(1-F\left(x_{i: m: n}\right)\right)^{R_{i}}\right) \\
& \times\left(1-F\left(x_{m: m: n}\right)\right)^{n-m-\sum_{i=1}^{J} R_{i}} \\
& 0<x_{1: m: n}<\cdots<x_{m: m: n}<\infty
\end{aligned}
$$

where

$$
d_{J}=\prod_{i=1}^{m}\left[n-i+1-\sum_{k=1}^{\max \{i-1, J\}} R_{k}\right] .
$$

In this paper, the underlying distribution is assumed to be the twoparameter generalized exponential distribution which is introduced by Gupta and Kundu (1999) as an alternative to the gamma and Weibull distributions. The two-parameter generalized exponential distribution has PDF and CDF are given, respectively, by

$$
\begin{aligned}
& f(x ; \alpha, \beta)=\frac{\alpha}{\beta} e^{-\frac{x}{\beta}}\left(1-e^{-\frac{x}{\beta}}\right)^{\alpha-1}, x>0, \\
& F(x ; \alpha, \beta)=\left(1-e^{-\frac{x}{\beta}}\right)^{\alpha}, x>0,
\end{aligned}
$$

where $\alpha>0$ and $\beta>0$ are the shape and scale parameters, respectively. This distribution has increasing and decreasing hazard rate depending on the shape parameter $\alpha$. Depending on $\alpha$, the generalized exponential distribution can be either reverse $J$-shaped or bell-shaped. If $\alpha<1$, it is reverse $J$-shaped while if $\alpha>1$, it is bell shaped. When $\alpha=1$, it becomes the exponential distribution. 
From (1), (3) and (4), we obtain the likelihood function of $\alpha$ and $\beta$, based on the adaptive Type-II progressively censored sample $\underline{\mathbf{X}}=$ $\left(X_{1: m: n}, \ldots, X_{m: m: n}\right)$ with censoring scheme $\underline{\mathbf{R}}=\left(R_{1}, \ldots, \overline{R_{m}}\right)$, as

$$
\begin{aligned}
L(\underline{\mathbf{x}} ; \theta, \sigma)= & d_{J} \prod_{i=1}^{m} \frac{\theta}{\sigma} e^{-\frac{x_{i}}{\sigma}}\left(1-e^{-\frac{x_{i}}{\sigma}}\right)^{\theta-1} \prod_{i=1}^{J}\left(1-\left(1-e^{-\frac{x_{i}}{\sigma}}\right)^{\theta}\right)^{R_{i}} \\
& \times\left(1-\left(1-e^{-\frac{x_{m}}{\sigma}}\right)^{\theta}\right)^{n-m-\sum_{i=1}^{J} R_{i}}
\end{aligned}
$$

where $d_{J}$ is defined in (2).

\section{THE POSTERIOR DISTRIBUTION}

In this section, we derive the joint and conditional posterior distributions of the two unknown parameters $\theta$ and $\sigma$. Under the assumption that both parameters $\theta$ and $\sigma$ are unknown and independent, we may consider the prior distributions of $\theta$ and $\sigma$ as independent gamma prior distributions, $\mathrm{G}(a, b)$ and $\mathrm{G}(c, d)$, respectively:

$$
\pi_{1}(c)=\frac{b^{a} c^{a-1} e^{-b c}}{\Gamma(a)}, \quad c>0
$$

and

$$
\pi_{2}(\beta)=\frac{\xi^{\gamma} \beta^{\gamma-1} e^{-\xi \beta}}{\Gamma(\gamma)}, \quad \quad \beta>0
$$

Multiplying $\pi_{1}(\theta \mid a, b)$ by $\pi_{2}(\sigma \mid c, d)$, we obtain the joint prior function of $\theta$ and $\sigma$ as

$$
\pi(\theta, \sigma)=\frac{b^{a} d^{c}}{\Gamma(a) \Gamma(c)} \theta^{a-1} \sigma^{c-1} \exp (-b \theta-d \sigma) .
$$

Using the likelihood function given in (5) and the joint prior given in (8), the joint posterior function of $\theta$ and $\sigma$ can be obtained as

$$
\begin{aligned}
\pi^{*}(\theta, \sigma \mid \underline{x}) \propto & \theta^{m+a-1} \sigma^{-m+c-1} e^{-b \theta-d \sigma}\left[\prod_{i=1}^{m} e^{-\frac{x_{i}}{\sigma}}\left(1-e^{-\frac{x_{i}}{\sigma}}\right)^{\theta-1}\right] \\
& \times\left[\prod_{i=1}^{J}\left(1-\left(1-e^{-\frac{x_{i}}{\sigma}}\right)^{\theta}\right)^{R_{i}}\right] \\
& \times\left(1-\left(1-e^{-\frac{x_{m}}{\sigma}}\right)^{\theta}\right)^{n-m-\sum_{i=1}^{J} R_{i}}
\end{aligned}
$$

From (9), the conditional posterior density function of $\theta$ given $\sigma$ can be obtained as

$$
\begin{aligned}
\pi_{\theta}^{*}(\theta \mid \sigma, \underline{\mathbf{x}}) \propto & \theta^{m+a-1} e^{-b \theta}\left[\prod_{i=1}^{m}\left(1-e^{-\frac{x_{i}}{\sigma}}\right)^{\theta-1}\right] \\
& \times\left[\prod_{i=1}^{J}\left(1-\left(1-e^{-\frac{x_{i}}{\sigma}}\right)^{\theta}\right)^{R_{i}}\right] \\
& \times\left(1-\left(1-e^{-\frac{x_{m}}{\sigma}}\right)^{\theta}\right)^{n-m-\sum_{i=1}^{J} R_{i}}
\end{aligned}
$$

Similarly, the conditional posterior density function of $\sigma$ given $\theta$ can be obtained as

$$
\begin{aligned}
\pi_{\sigma}^{*}(\sigma \mid \theta, \underline{\mathbf{x}}) \propto & \sigma^{-m+c-1} e^{-d \sigma}\left[\prod_{i=1}^{m} e^{-\frac{x_{i}}{\sigma}}\left(1-e^{-\frac{x_{i}}{\sigma}}\right)^{\theta-1}\right] \\
\times & {\left[\prod_{i=1}^{J}\left(1-\left(1-e^{-\frac{x_{i}}{\sigma}}\right)^{\theta}\right)^{R_{i}}\right] } \\
& \times\left(1-\left(1-e^{-\frac{x_{m}}{\sigma}}\right)^{\theta}\right)^{n-m-\sum_{i=1}^{J} R_{i}} .
\end{aligned}
$$

Unfortunately, the conditional posterior distribution of $\theta$ and $\sigma$ in (10) and (11) cannot be reduced analytically to well known distributions and therefore it is not possible to sample directly by standard methods, but the plot of it show that it is similar to normal distribution. So, to generate random numbers from these distributions, we use the M-H algorithm within the Gibbs Sampling scheme with normal proposal distribution.

\section{TWO-SAMPLE BAYESIAN PREDICTION FOR FUTURE ORDER STATISTICS}

One of the main objectives of statistical modeling is to predict future observations on the basis of available information and Bayesian methodology provides a natural way. Several authors addressed the issue of prediction and obtained the predictive bounds for future data. The aim of this section is to discuss the problem of predicting unobserved order statistics from the future sample from the generalized exponential distribution on the basis of an observed (informative) adaptive Type-II progressive censored data from the same distribution. The predictive density and distribution functions are obtained and used to determine prediction intervals for the unobserved order statistics. We consider the prediction problem in terms of the estimation of the posterior predictive density of a future observation for two-sample prediction case. We also construct predictive interval for the observed order statistics observation using Gibbs sampling procedure.

Suppose that $X_{1: m: n}^{R}, X_{2: m: n}^{R}, \ldots, X_{m: m: n}^{R}$ is the observed adaptive Type-II progressively censored sample drawn from the generalized exponential distribution and $Y_{1: N}, Y_{2: N}, \ldots, Y_{N: N}$ are unobserved order statistics from an independent future random sample (of size $N$ ) from the same distribution. Based on the observed adaptive Type-II progressively censored sample, our aim is to construct Bayesian prediction for the $s^{t h}$ order statistics, $Y_{s: N}, 1<s<N$. The marginal density function of $Y_{s: N}$, given $\theta>0$ and $\sigma>0$, is of the form:

$g_{(s)}\left(y_{s} \mid \theta, \sigma\right)=D(s) f\left(y_{s} \mid \theta, \sigma\right)\left[F\left(y_{s} \mid \theta, \sigma\right)\right]^{s-1}\left[1-F\left(y_{s} \mid \theta, \sigma\right)\right]^{n-s}$,

where $D(s)=\frac{n !}{(n-s) !(s-1) !}$

By substituting from (3) and (4) into $(12$, we obtain the marginal density and distribution functions of $Y_{s: N}$, respectively, as

$$
\begin{aligned}
g_{(s)}\left(y_{s} \mid \theta, \sigma\right) & =D(s) \frac{\theta}{\sigma} e^{-\frac{y_{s}}{\sigma}}\left(1-e^{-\frac{y_{s}}{\sigma}}\right)^{\theta s-1}\left[1-\left(1-e^{-\frac{y_{s}}{\sigma}}\right)^{\theta}\right]^{n-s} \\
& =D(s) \frac{\theta}{\sigma} e^{-\frac{y_{s}}{\sigma}} \sum_{k=o}^{n-s} a_{k}(s)\left(1-e^{-\frac{y_{s}}{\sigma}}\right)^{\theta(k+s)-1}
\end{aligned}
$$


and

$$
G_{(s)}\left(y_{s} \mid \theta, \sigma\right)=D(s) \sum_{k=o}^{n-s} a_{k}(s)\left[\frac{\left(1-e^{-\frac{y_{s}}{\sigma}}\right)^{\theta(k+s)}}{k+s}\right],
$$

where $a_{k}(s)=(-1)^{k}\left(\begin{array}{c}n-s \\ k\end{array}\right)$.

The Bayesian predictive density function of $Y_{s: N}$, based on the observed adaptive Type-II progressively censored sample, can be obtained as

$$
g_{(s)}^{*}\left(y_{s} \mid \theta, \sigma\right)=\int_{0}^{\infty} \int_{0}^{\infty} g_{(s)}\left(y_{s} \mid \theta, \sigma\right) \Pi^{*}(\theta, \sigma \mid \underline{x}) d \theta d \sigma,
$$

where $\Pi^{*}(\theta, \sigma \mid \underline{x})$ is the joint posterior density of $\theta$ and $\sigma$ as given in 9. Thus, the predictive distribution function, based on the observed adaptive Type-II progressively censored sample, is given by

$$
G_{(s)}^{*}\left(y_{s} \mid \theta, \sigma\right)=\int_{0}^{\infty} \int_{0}^{\infty} G_{(s)}\left(y_{s} \mid \theta, \sigma\right) \Pi^{*}(\theta, \sigma \mid \underline{x}) d \theta d \sigma .
$$

It is clear that $g_{(s)}^{*}\left(y_{s} \mid \theta, \sigma\right)$ in 15, , and $G_{(s)}^{*}\left(y_{s} \mid \theta, \sigma\right)$ in 16 cannot be expressed in closed form and hence it cannot be evaluated analytically. A simulation based on consistent estimator $g_{(s)}^{*}\left(y_{s} \mid \theta, \sigma\right)$, and $G_{(s)}^{*}\left(y_{s} \mid \theta, \sigma\right)$ can be obtained by using the MCMC-Gibbs sampling procedure with $\mathrm{M}-\mathrm{H}$, which is introduced by Metropolis et al. (1953) and then it is extended by Hastings (1970). We propose the following scheme to generate $\theta$ and $\sigma$ from the posterior density functions and in turn obtain the simulation estimators of the predictive density and distribution functions, $g_{(s)}^{*}\left(y_{s} \mid \theta, \sigma\right)$ and $G_{(s)}^{*}\left(y_{s} \mid \theta, \sigma\right)$, for $Y_{s: N}$.

(1) Start with an $\left(\theta^{(0)}, \sigma^{(0)}\right)$.

(2) Set $i=1$.

(3) Generate $\theta^{(i)}$ from $\pi_{\theta}^{*}(\theta \mid \sigma, \underline{\mathbf{x}})$ with the $N\left(\theta^{(i-1)}, \operatorname{Var}(\hat{\theta})\right)$ proposal distribution.

(4) Generate $\sigma^{(i)}$ from $\pi_{\sigma}^{*}(\sigma \mid \theta, \underline{\mathbf{x}})$ with the $N\left(\sigma^{(i-1)}, \operatorname{Var}(\hat{\sigma})\right)$ proposal distribution.

(5) Compute $\theta^{(i)}$ and $\sigma^{(i)}$.

(6) Set $i=i+1$

(7) Repeat steps $3-6 \quad N_{1}$ times.

(8) The simulation estimators of the predictive density and distribution functions, $g_{(s)}^{*}\left(y_{s} \mid \theta, \sigma\right)$ and $G_{(s)}^{*}\left(y_{s} \mid \theta, \sigma\right)$, for $Y_{s: N}$ can be obtained, respectively, as:

$$
g_{(s)}^{*}\left(y_{s} \mid \theta, \sigma\right)=\frac{1}{N_{1}-M_{1}} \sum_{i=M_{1}+1}^{N_{1}} g_{(s)}\left(y_{s} \mid \theta_{i}, \sigma_{i}\right)
$$

and

$$
G_{(s)}^{*}\left(y_{s} \mid \theta, \sigma\right)=\frac{1}{N_{1}-M_{1}} \sum_{i=M_{1}+1}^{N_{1}} G_{(s)}\left(y_{s} \mid \theta_{i}, \sigma_{i}\right) .
$$

where $M_{1}$ is burn-in.

Moreover, a symmetric $100(1-\gamma) \%$ predictive interval for $Y_{s: N}$, $s=1,2, \ldots, N$, can be obtained by solving the two following nonlinear equations

$$
P\left[Y_{s}>L \mid \underline{x}\right]=\frac{\gamma}{2}=1-\hat{G}_{(s)}^{*}\left(y_{s} \mid \theta, \sigma\right) \Longrightarrow \hat{G}_{(s)}^{*}\left(y_{s} \mid \theta, \sigma\right)=1-\frac{\gamma}{2},
$$

and

$P\left[Y_{s}>U \mid \underline{x}\right]=1-\frac{\gamma}{2}=1-\hat{G}_{(s)}^{*}\left(y_{s} \mid \theta, \sigma\right) \Longrightarrow \hat{G}_{(s)}^{*}\left(y_{s} \mid \theta, \sigma\right)=\frac{\gamma}{2}$,

where $L$ and $U$ are the lower and upper bounds of the predictive interval.

\section{NUMERICAL RESULTS}

\subsection{Simulation study}

A simulation study is carried out for evaluating the performance of the inferential methods discussed in the paper. We chose $n=30$, $m=10$ and $R=(3,2,3,1,2,3,1,0,2,3)$. For $T=0.8$, we first describe the algorithm, proposed by $\mathrm{Ng}$ et al. (2009), to generate adaptive Type-II progressively censored sample from the generalized exponential distribution with parameters $(\theta, \sigma)=(2,3)$.

(1) Generate an ordinary Type-II progressively censored sample $X_{1, m, n}, \ldots, X_{m, m, n}$ with the given censoring scheme $R=$ $\left(R_{1}, \ldots, R_{m}\right)$ from the GE distribution using the proposed algorithm in Balakrishnan and Sandhu (1995).

(2) Determine the value of $J$, where $X_{J: m: n}<T<X_{J+1: m: n}$, and discard the sample $X_{J+2, m, n}, \ldots, X_{m, m, n}$.

(3) Generate the first $m-J-1$ order statistics from a truncated distribution $f(x) /\left[1-F\left(x_{j+1: m: n}\right)\right]$ with sample size $(n-$ $\left.\sum_{i=1}^{J} R_{i}-J-1\right)$ as $X_{j+2: m: n}, X_{j+3: m: n}, \ldots, X_{m: m: n}$, where $f(x)$ and $F(x)$ are given in (2.3) and (2.4), respectively.

We used the above algorithm to generate adaptive Type-II progressively censored sample and then computed the lower and upper bounds of the $100(1-\gamma) \%$ predictive interval of the order statistic $Y_{s: N}, s=1,2, \ldots, N$, from a future random sample of size $N=5$ based on $\left(N_{1}=11000\right)$ MCMC samples and discard the first $\left(M_{1}=1000\right)$ values as burn-in. We replicated the process 1000 times and compute the average values of the lower bound, upper bound and width of the $100(1-\gamma) \%$ predictive interval when $\gamma=0.01,0.05,0.10,0.20$. The results are obtained using the non-informative gamma priors for the two parameters with $a=b=c=d=0$ (we call it MCMC - prior 0) and the informative gamma priors for the two parameters with $a=1, b=2, c=1$ and $d=2$ (we call it MCMC - prior 1). The obtained results are presented in Tables 1-4.

From the results obtained in Tables 1-4, it can be observed that the Bayesian prediction intervals with informative priors are tighter than the width of those with noninformative priors. Also, when the significant level $(\gamma)$ increases, the width of the prediction interval decreases in all cases. Moreover, the width of all prediction intervals increase with increasing $s$.

\subsection{Numerical example}

To illustrate the inferential procedures developed in the preceding sections, we consider the following data giving the number of million revolutions before failure for each of 23 ball bearings in the life test. These data are taken from Lawless (1982, Page 228) and has been used earlier by Gupta and Kundu (2002)

$\begin{array}{llllllll}17.88 & 28.92 & 33.00 & 41.52 & 42.12 & 45.60 & 48.80 & 51.84\end{array}$ $\begin{array}{llll}51.96 & 54.12 & 55.56 & 67.80\end{array}$

$\begin{array}{llllllll}68.64 & 68.64 & 68.88 & 84.12 & 93.12 & 98.64 & 105.12 & 105.84\end{array}$ $\begin{array}{lll}127.92 & 128.04 & 173.40\end{array}$

In this example, we use this set of real data to generate an adaptive Type-II progressively censored sample with $n=23, m=10$, 
$T=45$ and $\mathrm{R}=(2,0,1,3,0,0,2,0,1,4)$. Thus, the generated adaptive Type-II progressively censored sample is

42.12 , 51.96, 54.12, 67.8, 68.64, 84.12, 93.12, 98.64, 105.12, $105.84,127.92,128.04,173.4$

Because we have no prior information about the unknown parameters, we assume here the non-informative prior (with $a=b=$ $c=d=0$ ). Based on the generated adaptive Type-II progressively censored sample, we compute the lower and upper bounds of the $100(1-\gamma) \%$ predictive interval of the order statistic $Y_{s: N}$, $s=1,2, \ldots, N$, from a future random sample of size $N=5$. The lower bound, upper bound and width of the $99 \%$ and $95 \%$ predictive intervals are presented in Table 5. The lower bound, upper bound and width of the $90 \%$ and $80 \%$ predictive intervals are presented in Table 6.

\subsection{Conclusion}

In this paper, we discussed the problem if predicting unobserved order statistics from a future sample based on observed adaptive Type-II progressively censored sample from the generalized distribution. We derived the joint and conditional posterior functions for the unknown parameters. Also, we used the MCMC-Gibbs sampling procedure with $\mathrm{M}-\mathrm{H}$ to estimate the predictive density and distribution functions of $Y_{s: N}$ and then calculated the Bayesian prediction interval of $Y_{s: N}, s=1, \ldots N$. From all obtained results in the simulation study and the numerical example, we can notice that the Bayesian prediction interval with informative prior is better than that with noninformative prior.

\section{REFERENCES}

1. Amein, M.M. (2016). Estimation for Unknown Parameters of the Burr Type-XII Distribution Based on an Adaptive Progressive Type-II Censoring Scheme. Journal of Mathematics and Statistics 12, 119-126.

2. Amein, M.M. (2017). Estimation for Unknown Parameters of the Extended Burr Type-XII Distribution Based on an Adaptive Type-II Progressive Censoring Scheme, Global Journal of Pure and Applied Mathematics 13, 7709-7724.

3. Balakrishnan, N. (2007). Progressive censoring methodology: An appraisal (with discussions). Test 16, 211-296.

4. Balakrishnan, N. and Aggarwala, R. (2000). Progressive Censoring: Theory, Methods, and Applications, Birkhauser, Boston, Berlin .

5. Bernardo, J.M. (1988). Bayesian linear probabilistic classifcation. In: Gupta, S.S., Berger, J.O. (Eds.), Statistical Decision Theory and Related Topics IV, vol. 1. Springer, Berlin, pp. 151162.

6. Brown, P.J. and Makalainen, T. (1992). Regression, sequential measurements and coherent calibration. In: Bernardo, J.M., Berger, J.O., Dawid, A.P., Smith, A.F.M. (Eds.), Bayesian Statistics 4. Oxford University Press, Oxford, pp. 97108 (with discussion).

7. Cramer, E. and Iliopoulos, G. (2010). Adaptive progressive Type-II censoring. Test 9, 342-358.

8. Devroye, L. and Gyorfi, L. (1985). Nonparametric Density Estimation: The $L_{1}$ View, Wiley, New York.

9. Gupta, R.D. and Kundu, D. (1999). Generalized exponential distribution. Australian \& New Zealand Journal of Statistics 41 173-188.

10. Hastings, W.K. (1970). Monte Carlo sampling methods using Markovchains and their applications. Biometrika 57, 97-109.
11. Lawless, J.F. (1982). Statistical models and methods for lifetime data. New York: Wiley.

12. Mahmoud, M.A.W., Soliman, A.A., Abd Ellah, A.H., ElSagheer, R.M. (2013). Estimation of Generalized Pareto under an Adaptive Type-II Progressive Censoring," Intelligent Information Management 5, 73 -83.

13. Metropolis, N., Rosenbluth, A. W., Rosenbluth, M. N., Teller, A. H. and Teller, E.(1953). Equations of State Calculations by fast Computing Machines, Journal Chemical Physics 21, 10871091.

14. Mohie El-Din, M. M. M., Amein, M. M., Shafay, A. R. and Mohamed, S. (2017). Estimation of generalized exponential distribution based on an adaptive progressively type-II censored sample, Journal of Statistical Computation and Simulation 87, 1292-1304.

15. Mohie El-Din, M.M. and Shafay, A.R. (2013). One- and TwoSample Bayesian Prediction Intervals Based on Progressively Type-II Censored Data. Statistical papers 54, 287-307.

16. Nelson, W. (1982). Applied life data analysis. NewYork: Wiley.

17. Ng, H.K.T and Chan, P.S. (2007). Discussion on Progressive censoring methodology: an appraisal by N. Balakrishnan. Test 16, 287-289.

18. Ng, H.K.T., Kundu, D. and Chan, P.S. (2009). Statistical Analysis of Exponential Lifetimes under an Adaptive Type-II Progressive Censoring Scheme. Naval Research Logistics 56, 687698. 
Table 1. The average values of the lower bound, upper bound and width of the $99 \%$ predictive interval for $Y_{s: 5}, s=1, \ldots, 5$.

\begin{tabular}{c|c|c|c|c|c}
\hline \hline \multicolumn{3}{c|}{$($ MCMC- prior 0) } & \multicolumn{3}{|c}{$($ MCMC-prior 1) } \\
\hline$Y_{s: 5}$ & $($ Lower, Upper $)$ & Width & $Y_{s: 5}$ & $($ Lower, Upper $)$ & Width \\
\hline$Y_{1: 5}$ & $(0.0399,10.4585)$ & 10.4186 & $Y_{1: 5}$ & $(0.1369,7.5849)$ & 7.4480 \\
\hline$Y_{2: 5}$ & $(0.2819,11.8369)$ & 11.5550 & $Y_{2: 5}$ & $(0.6881,10.5882)$ & 9.9001 \\
\hline$Y_{3: 5}$ & $(1.3206,18.6678)$ & 17.3471 & $Y_{3: 5}$ & $(1.3285,15.3512)$ & 14.0227 \\
\hline$Y_{4: 5}$ & $(2.4704,44.7430)$ & 42.2726 & $Y_{4: 5}$ & $(2.2304,22.5209)$ & 20.2905 \\
\hline$Y_{5: 5}$ & $(3.9501,47.4535)$ & 43.5034 & $Y_{5: 5}$ & $(3.6449,39.2798)$ & 35.6349 \\
\hline \hline
\end{tabular}

Table 2. The average values of the lower bound, upper bound and width of the $95 \%$ predictive interval for $Y_{s: 5}, s=1, \ldots, 5$.

\begin{tabular}{c|c|c|c|c|c}
\hline \hline \multicolumn{3}{c|}{$($ MCMC-prior 0$)$} & \multicolumn{3}{|c}{$($ MCMC- prior 1$)$} \\
\hline$Y_{s: 5}$ & $($ Lower, Upper $)$ & Width & $Y_{s: 5}$ & $($ Lower, Upper $)$ & Width \\
\hline$Y_{1: 5}$ & $(0.2787,7.7365)$ & 7.4578 & $Y_{1: 5}$ & $(0.2812,5.9442)$ & 5.6630 \\
$Y_{2: 5}$ & $(1.2391,9.5651)$ & 8.3259 & $Y_{2: 5}$ & $(1.1009,8.4139)$ & 7.3130 \\
$Y_{3: 5}$ & $(2.1861,13.6587)$ & 11.4726 & $Y_{3: 5}$ & $(2.077,11.9393)$ & 9.8622 \\
$Y_{4: 5}$ & $(3.5211,26.4485)$ & 22.9274 & $Y_{4: 5}$ & $(3.1976,17.2451)$ & 14.0474 \\
$Y_{5: 5}$ & $(4.7753,37.5113)$ & 32.7359 & $Y_{5: 5}$ & $(5.0236,32.3138)$ & 27.2901 \\
\hline \hline
\end{tabular}

Table 3. The average values of the lower bound, upper bound and width of the $90 \%$ predictive interval for $Y_{s: 5}, s=1, \ldots, 5$.

\begin{tabular}{c|c|c|c|c|c}
\hline \hline \multicolumn{3}{c|}{$($ MCMC- prior 0) } & \multicolumn{3}{|c}{$($ MCMC-prior 1) } \\
\hline$Y_{s: 5}$ & $($ Lower, Upper $)$ & Width & $Y_{s: 5}$ & $($ Lower, Upper $)$ & Width \\
\hline$Y_{1: 5}$ & $(0.2116,6.9398)$ & 6.7282 & $Y_{1: 5}$ & $(0.4814,5.0934)$ & 4.6120 \\
$Y_{2: 5}$ & $(1.4914,9.2594)$ & 7.7680 & $Y_{2: 5}$ & $(1.4883,7.3580)$ & 5.8697 \\
$Y_{3: 5}$ & $(2.409,10.4310)$ & 8.0220 & $Y_{3: 5}$ & $(2.6075,9.5793)$ & 6.9718 \\
$Y_{4: 5}$ & $(4.0827,20.2031)$ & 16.1204 & $Y_{4: 5}$ & $(3.5975,13.8451)$ & 10.2475 \\
$Y_{5: 5}$ & $(6.1443,29.8928)$ & 23.7485 & $Y_{5: 5}$ & $(5.4645,22.5461)$ & 17.0816 \\
\hline \hline
\end{tabular}

Table 4. The average values of the lower bound, upper bound and width of the $80 \%$ predictive interval for $Y_{s: 5}, s=1, \ldots, 5$.

\begin{tabular}{c|c|c|c|c|c}
\hline \hline \multicolumn{3}{c|}{$($ MCMC- prior 0) } & \multicolumn{3}{|c}{$($ MCMC-prior 1) } \\
\hline$Y_{s: 5}$ & $($ Lower, Upper $)$ & Width & $Y_{\text {s:5 }}$ & $($ Lower, Upper $)$ & Width \\
\hline$Y_{1: 5}$ & $(0.5926,5.0386)$ & 4.4460 & $Y_{1: 5}$ & $(0.8192,4.2796)$ & 3.4604 \\
$Y_{2: 5}$ & $(1.8149,8.5751)$ & 6.7602 & $Y_{2: 5}$ & $(1.8038,6.3711)$ & 4.5673 \\
$Y_{3: 5}$ & $(3.119,10.2792)$ & 7.1601 & $Y_{3: 5}$ & $(2.9292,8.8177)$ & 5.8885 \\
$Y_{4: 5}$ & $(4.6125,13.3709)$ & 8.7584 & $Y_{4: 5}$ & $(4.3643,12.0921)$ & 7.7277 \\
$Y_{5: 5}$ & $(6.7396,20.8389)$ & 14.0993 & $Y_{5: 5}$ & $(6.4141,19.7169)$ & 13.3027 \\
\hline \hline
\end{tabular}

Table 5. The average values of the lower bound, upper bound and width of the $99 \%$ and $95 \%$ predictive interval for $Y_{s: 5}, s=1, \ldots, 5$.

\begin{tabular}{c|c|l|l|l|l}
\hline \hline \multicolumn{3}{c|}{$99 \%$ predictive intervals } & \multicolumn{3}{|c}{$95 \%$ predictive intervals } \\
\hline$Y_{s: 5}$ & (Lower, Upper $)$ & Width & $Y_{s: 5}$ & (Lower, Upper $)$ & Width \\
\hline$Y_{1: 5}$ & $(5.3360,88.9052)$ & 83.5692 & $Y_{1: 5}$ & $(10.7497,72.2713)$ & 61.5216 \\
$Y_{2: 5}$ & $(19.2642,109.4150)$ & 90.1511 & $Y_{2: 5}$ & $(21.5242,100.3500)$ & 78.8256 \\
$Y_{3: 5}$ & $(24.9427,153.6400)$ & 128.6970 & $Y_{3: 5}$ & $(32.8750,126.6540)$ & 93.7792 \\
$Y_{4: 5}$ & $(36.2476,226.0080)$ & 189.7600 & $Y_{4: 5}$ & $(44.9061,164.7790)$ & 119.873 \\
$Y_{5: 5}$ & $(49.3578,336.7620)$ & 287.4040 & $Y_{5: 5}$ & $(63.9343,337.0110)$ & 273.077 \\
\hline \hline
\end{tabular}

Table 6. The average values of the lower bound, upper bound and width of the $90 \%$ and $80 \%$ predictive interval for $Y_{s: 5}, s=1, \ldots, 5$.

\begin{tabular}{c|c|c|c|c|c}
\hline \hline \multicolumn{5}{c|}{$90 \%$ predictive intervals } & \multicolumn{3}{|c}{$80 \%$ predictive intervals } \\
\hline$Y_{s: 5}$ & $($ Lower, Upper $)$ & Width & $Y_{s: 5}$ & $($ Lower, Upper $)$ & Width \\
\hline$Y_{: 5}$ & $(13.2100,65.5197)$ & 52.3097 & $Y_{1: 5}$ & $(17.4271,56.9228)$ & 39.4957 \\
$Y_{2: 5}$ & $(26.1831,91.8664)$ & 65.6833 & $Y_{2: 5}$ & $(30.4093,77.9610)$ & 47.5517 \\
$Y_{3: 5}$ & $(37.3926,124.9530)$ & 87.5600 & $Y_{3: 5}$ & $(44.1026,99.6267)$ & 55.5242 \\
$Y_{4: 5}$ & $(50.7692,159.5960)$ & 108.8270 & $Y_{4: 5}$ & $(56.3729,124.2150)$ & 67.8418 \\
$Y_{5: 5}$ & $(61.5428,222.2450)$ & 221.2450 & $Y_{5: 5}$ & $(73.3478,235.4770)$ & 162.1290 \\
\hline \hline
\end{tabular}

